\title{
CLASSICAL IMPACT DAMPER AND PENDULUM IMPACT DAMPER FOR POTENTIAL CIVIL ENGINEERING APPLICATION
}

\author{
By George Sy CHUA*, Benito M. PACHECO**, Yozo FUJINO*** and Manabu ITO****
}

\begin{abstract}
The classical impact damper (CID) and the pendulum impact damper (PID) are studied for the suppression of horizontal vibrations of civil engineering structures. Experiments on the PID to verify the validity of the mathematical model are done and comparisons with simulation results are provided. Parametric study under harmonic excitation shows that the effectiveness of the CID is sensitive to changes in the amplitude of the excitation. On the other hand, the PID is insensitive to changes in the amplitude of the excitation when its frequency is close to the structural frequency, and it is less sensitive to deviations in tuning than the tuned mass damper (TMD). Comparison of the CID, PID and TMD shows optimum efficiency in terms of mass ratio, under steadystate motion and ideal conditions, to be highest for the TMD and lowest for the PID. Included is a discussion of the practical constraints that may govern the actual choice of damper.

Keywords : civil engineering structures, classical impact damper, experiment, horizontal vibration, pendulum impact damper, restitution. simulation, steady-state motion, tuned mass damper
\end{abstract}

\section{INTRODUCTION}

Impact dampers utilize impacts occurring between damper mass and its container as the main mechanism for dissipating vibration energy (Fig. 1). It has been studied from as early as the $1940 \mathrm{~s}^{1)}$, but studies were mainly for mechanical systems ${ }^{1 / 10)}$, i. e., conditions in machines. For example, operating frequency was relatively fixed and large ratio of damper mass to machine mass was feasible. Many studies limit their investigation to conditions of two equally-spaced impacts per cycle (2 EIPC) ${ }^{2)-5)}$. This is because Lieber and Jensen" showed theoretically that maximum energy is dissipated in 2 EIPC motion. More recent studies $^{(6)}{ }^{7)}$ considering general impact condition showed it is beneficial to have 2 EIPC. This is based on numerical and experimental studies showing maximum reduction in displacement amplitude at resonant condition with 2 EIPC. Other studies extended the 2 EIPC condition by removing the symmetry criteria, i. e., two impacts per cycle (2 IPC) but not necessarily at equal time intervals ${ }^{8) \sim 10)}$. Due to the limitations set on the impact condition, results of these studies show only the performance of the impact damper under 2 EIPC or 2 IPC. For machines whose excitation frequency and amplitude are relatively fixed and known in magnitude, knowing the region where optimal performance occurs is sufficient for design purposes. With the impact damper properly designed, the probability of system parameters varying beyond 2 EIPC or 2 IPC condition is considered minimal. In contrast, under civil engineering conditions where excitation frequency and amplitude vary over a wider range, civil engineers are concerned about how the damper

\footnotetext{
* Member of JSCE, Dr. Eng. TYK Corp., Res. and Dev. Center (3-1 Ohbata-cho, Tajimi City, Gifu Pref. 507)

** Member of JSCE, Dr. Eng., Asso. Prof., Dept. of Civil Eng., Univ. of Tokyo (Bunkyo-ku, Tokyo 113)

*** Member of JSCE, Ph. D., Asso. Prof. , ditto

**** Member of JSCE, Dr. Eng., Prof., ditto
} 


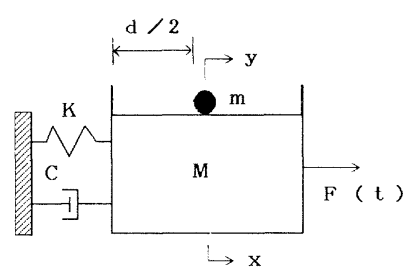

(a) Classical

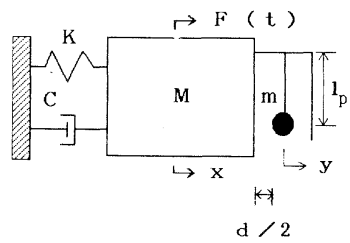

(b) Pendulum

Fig. 1 Examples of impact damper.

performance changes as impact condition deviates from 2 EIPC or 2 IPC condition.

Recently, experimental studies for specific civil engineering application were done ${ }^{(1)}{ }^{12)}$ that showed reduction in response amplitude of as much as $50 \%$ for a $4 \%$ mass ratio. To complement such studies, the present work provides analysis of two types of impact dampers, considering a wide range of parameters that are deemed representative of many civil engineering structures. Both the classical impact damper (CID) and a modified configuration in the form of the pendulum impact damper (PID) are studied. The PID can be looked at as a simplified model of the chain impact damper ${ }^{3)}{ }^{11}$. The PID would be less affected by rusting or geometric imperfections that could trouble the CID. Experiments on the PID are performed to verify the validity of the mathematical model. Experiments and numerical simulations on the PID have not been done before. A comparison of performance among the CID, PID and tuned mass damper (TMD) is provided to give a better idea of the effectiveness of the impact dampers.

It should be kept in mind, however, that certain physical and practical considerations may pose limits on the actual implementation of the impact dampers. For example, availability of space in the main system imposes a limit on the maximum size of the damper mass and the clearance that can be provided between the damper mass and its container. Such constraints are also discussed.

\section{THEORETICAL AND NUMERICAL ANALYSIS}

The present analysis is done under the following conditions. Parameter values and ranges are chosen to suit civil engineering conditions. Impact is not necessarily at every half cycle of structural vibration. The exciting force inducing horizontal vibration is harmonic. Non-harmonic forces should be considered in future studies, as they are frequently encountered in civil engineering structures. The structure is modelled as a linear single-degree-of-freedom (SDOF) system. Multi-degree-of- freedom (MDOF) model may be necessary when two or more modes of the structure are strongly interacting.

\section{(1) Parameters}

The following is a list of the parameters involved in the analysis and design of the CID and PID.

a) mass ratio, $\mu=m / M$ : ratio of the damper mass over the main system mass.

b) structural damping ratio, $\beta$ : inherent damping of the main system before attachment of the damper.

c) coefficient of restitution, $e:$ a number between zero and one, inclusive, which gives the relation of velocities just before and just after collision of two free masses.

d) gap size, $d:$ the total clearance between the damper mass and the walls of the container.

e) frequency ratio, $r=\Omega / \omega:$ ratio of the frequency of excitation over the frequency of the main system.

f) amplitude of excitation, $F_{0}$.

g) tuning ratio, $r^{\prime}=\omega_{p} / \omega:$ ratio of the frequency of the pendulum over that of the main system. Tuned condition refers to a tuning ratio equal to 1.0. Undertuned condition refers to a tuning ratio less than 1.0 while overtuned condition refers to a tuning ratio greater than 1.0. 
The equations given below were derived by Masri2) for steady-state 2 EIPC motion of the SDOF system. The assumptions include instantaneous impacts and frictionless contacts. The equations of motion between impacts are:

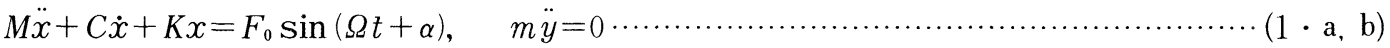

where $M, C, K=$ mass, damping and stiffness coefficients of main system, $F_{0}, \Omega=$ amplitude and frequency of exciting force, $\alpha=$ phase angle, $t=$ time measured from last impact, $x, y=$ absolute displacement coordinates of main system and damper mass, $m=$ mass of damper, ' , ' first and second derivatives with respect to time.

The complete closed-form solution of Eq. $(1 \cdot a)$ for motion between impacts is :

$x=\exp ^{-\beta \omega t}\left(B_{1} \sin \eta \omega t+B_{2} \cos \eta \omega t\right)+A \sin (\Omega t+\tau)$ for $0 \leqq \Omega t \leqq \pi \cdots$

where $\quad \beta=C /(2 M \omega), \quad \omega=\sqrt{K / M}, \quad \eta=\sqrt{1-\beta^{2}}, \quad A=\left(F_{0} / K\right) / \sqrt{\left.1-r^{2}\right)^{2}+(2 \beta r)^{2}}$

$$
\begin{aligned}
& B_{1}=(d / 2)\left(1+h_{2}\right)\left(\sigma_{2}-\sigma_{1}\right) Q / \Delta, \quad B_{2}=(d / 2) h_{1}\left(\sigma_{1}-\sigma_{2}\right) Q / \Delta \\
& r=\tan ^{-1}\left[\left(-2 \rho \pm H \sqrt{H^{2}+4-\rho^{2}}\right)\right] /\left[\left(-\rho h 2 \sqrt{H^{2}+4-\rho^{2}}\right)\right] \\
& \Delta=h_{1}\left[Q\left(\sigma_{2}-\sigma_{1}\right)-\left(S+Q \sigma_{2}\right) \sigma_{1} \theta_{2}+\left(S+Q \sigma_{1}\right) \beta \omega \sigma_{2}\right]+\left(1+h_{2}\right)\left[\left(S+Q \sigma_{2}\right) \sigma_{1} \theta_{1}+\left(S+Q \sigma_{1}\right) \eta \omega \sigma_{2}\right] \\
& h_{1}=\exp (-\beta \pi / r) \sin (\eta \pi / r) . \quad h_{2}=\exp (-\beta \pi / r) \cos (\eta \pi / r) \\
& \sigma_{1}=(\pi / 2 \Omega)(1+e) /(1-e+2 \mu), \quad \sigma_{2}=(\pi / 2 \Omega)(1+e) /(1-e-2 \mu e) \\
& \theta_{1}=-\beta \omega h_{1}+\eta \omega h_{2} \text {, } \\
& S=\sin (\tau), \quad Q=\Omega \cos (\tau), \rho=d / A \\
& H=2 \Omega\left\{h_{1}\left[\left(\sigma_{2}-\sigma_{1}\right)+\sigma_{1} \sigma_{2}\left(\beta \omega-\theta_{2}\right)\right]+\left(1+h_{2}\right)\left[\sigma_{1} \sigma_{2}\left(\theta_{1}+\eta \omega\right)\right]\right\} / \\
& \left\{h_{1}\left[\beta \omega \sigma_{2}-\sigma_{1} \theta_{2}\right]+\left(1+h_{2}\right)\left[\sigma_{1} \theta_{1}+\eta \omega \sigma_{2}\right]\right\}
\end{aligned}
$$

(Note that the factor $\sigma_{1} \theta_{2}$ in the equation for $\Delta$ was written as $\sigma_{1} \theta_{1}$ in Masri's paper.) The two sets of signs appearing in the equation for $\tau$ given above, correspond to two distinct steady-state solutions. The analytical criteria to decide which of the solutions is valid, if any, is furnished by the stability analysis of the solutions. The procedure for the stability analysis involves introducing a small perturbation to the solution immediately after an impact, and determining the deviation of the perturbed solution from the non-perturbed solution right after the next impact. Repeating the process, the effect of the initial perturbation on the steady-state solution after any number of impacts could be determined. Stability of the solution is established by showing that the deviation decays as the number of successive impacts considered is increased. This has been shown mathematically by Masri ${ }^{2}$.

Varying $\Omega t$ from 0 to $\pi$ using fine steps in Eq. (2), or differentiating Eq. (2) with respect to $t$, the steady-state displacement amplitude of the main system with CID could be obtained.

Since Eq. (2) is valid only for 2 EIPC condition, numerical time integration is utilized to obtain solutions for non-2 EIPC conditions. During impact, assumed to occur instantaneously when abs $(y-x)=$ $d / 2$, the combined momentum equation and restitution relation are utilized to obtain the relationship of velocities just before and just after the impact. The relations are:

$$
\dot{x}_{+}=(1-\mu e) /(1+\mu) \dot{x}_{-}+\mu(1+e) /(1+\mu) \dot{y}_{-}, \quad \dot{y}_{+}=(1+e) /(1+\mu) \dot{x}_{-}+(\mu-e) /(1+\mu) \dot{y}_{-}
$$

where - , + subscripts refer to just before and just after impact conditions.

As a check for correctness of both the closed-form equation (Eq. (2)) and the numerical time integration procedure used, simulations using parameters that give 2 EIPC were done. The results show that amplitudes given by both procedures are practically equal, except at the limits of the range of 2 EIPC. When differences occur at the limits, the closed-form solution value is utilized.

(3) Pendulum impact damper

Fig. 1 ( b ) shows the mathematical model. The PID is represented as a container rigidly attached to the main structure with the PID mass suspended from the center of the container. Assuming small displacement amplitudes for the pendulum, the equations of motion between impacts are:

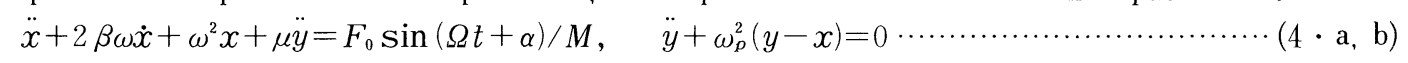


where $\omega_{p}=\sqrt{r-l_{p}}=$ natural frequency of pendulum, $r=$ acceleration of gravity, $l_{p}=$ length of the pendulum, and other variables as defined for Eqs. (1) and (2).

Impacts are treated in the same way as for the CID (Eqs. (3)).

\section{EXPERIMENTS ON THE PENDULUM IMPACT DAMPER}

These experiments on the PID are done to verify the validity of the mathematical model and to investigate the effect of using different wall materials on the noise produced during impacts.

\section{(1) Experiment model}

Fig. 2 shows the experiment model which is composed of 2 steel plates as the cantilevered member, a base plate to attach the PID, and 2 steel angles as walls of the PID container. The mass of the structure is adjusted by adding masses on the base plate to give a natural frequency of $1.5 \mathrm{~Hz}$. The structure is intended to have very low inherent damping to enable accurate measurements of additional damping.

The pendulum masses used are commercially available steel balls of diameters 30,40 and $50 \mathrm{~mm}$. The pendulum is hung from hooks provided under the base plate. Tuning of the pendulum is achieved by changing the hanging length of the steel ball. Gap size is adjusted by moving the two angles to equal distances from the center of the base plate.

A magnetic excitor constructed and used as the source of horizontal harmonic excitation is composed of a rectangular cardboard tube, rigidly fixed to an external support, where the center half of the tube is wrapped with magnetic coils. Permanent magnets are rigidly fixed on the legs of a U-bar that is attached to the structure. A frequency wave generator sends sinusoidal current through the magnetic coil, which then interacts with the magnetic field between the permanent magnets. This produces an excitation force that does not inhibit motion of the structure because there are no solid connections between the structure and the excitor.

The mass and natural frequency $(1.5 \mathrm{~Hz})$ of the structure are fixed while the inherent damping is measured through both free and forced vibration experiments. (Details of procedure are in the next section.)

Fig. 3 (a) shows $\delta$ (logarithmic decrement) versus displacement amplitude plot of the structure (without damper) for free vibration. The average $\delta$ is 0.003 . It may be noted that there are no experiment

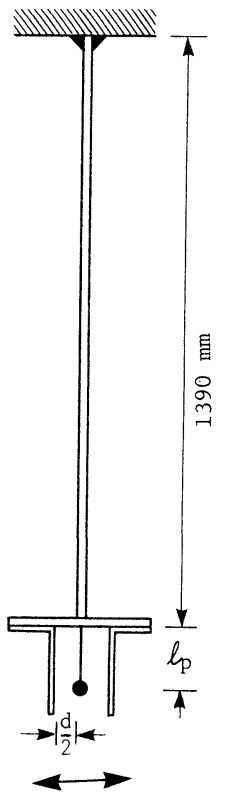

Fig. 2 Experiment model.

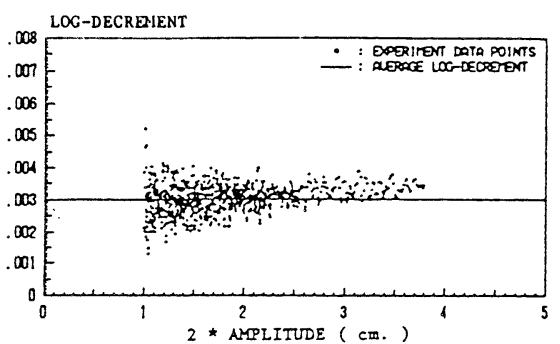

(a) Free vibration

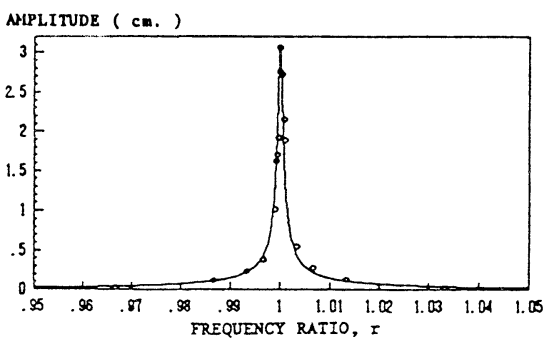

(b) Forced vibration

Fig. 3 Vibration property of main structure without PID. 
data points plotted for double amplitudes lower than $1.0 \mathrm{~cm}$. This is because there is a large scatter of the experiment data points, but the averaged $\delta$ for these points is 0.003 .

Fig. 3 ( b ) shows the frequency response curve of the structure (without damper) for forced vibration. $\beta=0.00048$ (damping ratio) is obtained using the half-power method and this gives $\delta=0.003$, agreeing with the free vibration result.

It can be observed from Fig. 3 that the simulation curves using $\beta=0.00048$ fit the experiment data points very well. Thus $\beta=0.00048$ for the structure is used in all simulations for comparison with results from experiment.

\section{(2) Experiment procedure}

Displacement of base plate is detected by an optical-type position sensor. The light-emitting target is attached to the base plate. The sensor camera is placed directly above this target, at sufficient height so that displacements of target up to $2 \mathrm{~cm}$ can be detected.

The data collected are digital record and pen-recorder plot of the history of displacement of base plate, manual record of structure vibration frequency and excitation frequency obtained from a frequency counter, and visual and audio observation of impacts manually marked on the pen-recorder plot ${ }^{13 .}$.

For free vibration, different initial displacements are given to the structure (10 to $25 \mathrm{~mm}$ ) to check that initial conditions do not affect the log-decrement $(\delta)$ value at a given amplitude. The instruments used also prevented one continuous record of displacement decay to be taken.

For forced vibration, a frequency sweep from $r=0.9$ to 1.1 is done to obtain the frequency response curve of the structure. Steady-state vibration amplitude is determined from the root-mean-square of the digital data points multiplied by the square-root of two. This is to enable comparison of amplitude between steady-state response where the successive peaks are equal (Fig. 4 (a)) with those where beating (Figs. 4 (b) and (c)) occurs. The frequency ratio range of $0.9<r<1.1$ is expected to contain the primary resonances of the structure with damper. Note from Fig. 3 ( b ) that the resonance of the structure without damper is contained in the narrower range of $0.99<r<1.01$.

The pendulum damping is assumed to be negligible. The other parameters varied are $\mu=0.54 \%$, $1.29 \%, 2.52 \%, d=20,30,40 \mathrm{~mm}, r^{\prime}=0.9,1.0,1.1$, and wall material $(e)$.

Values of $e$ for the different materials used are experimentally determined. The procedure adopted is to drop the steel balls from heights of 25,50 , and $100 \mathrm{~cm}$ on to the steel angle clamped to the structure. This is taped on video for slow motion playback. $e$ is computed utilizing Eq. (3 $\cdot$ b) with $\dot{x}_{-}=0.0$ (structure stationary before and after the impact), $\dot{y}_{-}$and $\dot{y}_{+}$are determined from the heights the steel ball drops from and rebounds to, respectively, and $\mu$ is known for each size of steel ball. Since this procedure incorporates some approximations, an average value of $e$ in terms of the different masses of steel balls and the velocities at impact is deemed more appropriate. The following are the average values obtained. Steel on steel $: e=0.37$. Steel on $0.5 \mathrm{~mm}$ rubber lining over steel $: e=0.58$. Steel on $1.0 \mathrm{~mm}$ rubber lining over steel $: e=0.46$. Steel on $3.0 \mathrm{~mm}$ cork lining over steel $: e=0.64^{133}$.

For free vibration, the approximate time intervals where one, two, three, etc. number of impacts per cycle is observed are marked on the pen-recorder plot of time history, while only regular impacts at steady-state is observed for forced vibration.

\section{( 3 ) Comparison of simulation with experiment}

Fig. 5 (a) shows a typical plot of free vibration delta $\delta$ (system with PID $\delta$ minus the structure $\delta=$ $0.003)$ versus displacement amplitude of the structure. The material in the experiment is steel ball impacting with the steel walls, thus $e=0.37$ is used for the simulation. Fig. 5 (b) shows a typical frequency response curve of the structure with the PID attached. The material in the experiment is steel ball impacting with the steel wall having a $1.0 \mathrm{~mm}$ rubber lining, thus $e=0.46$ is used for the simulation (Not too many cases with different values of frequency, ratio, $r$, have been simulated, because each simulation is very time consuming. The resolution of $\Delta r=0.01$ in Fig. 5 ( b ) is considered sufficient 

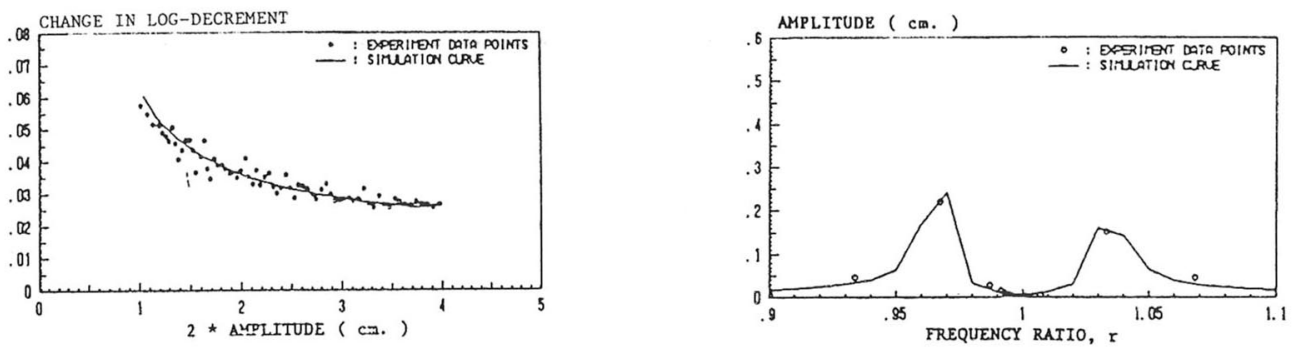

Fig. 5 Vibration property of main structure with PID.

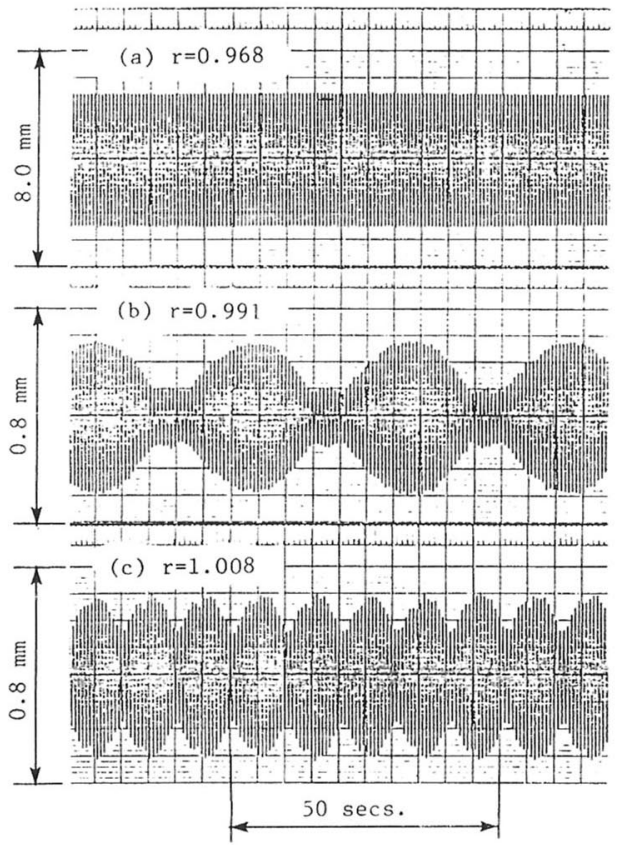

Fig. 4 Sample time history from experiment

here, but in parametric studies in section 5 below, a finer resolution of $\Delta r=0.005$ is used). It can be observed here that good fit exists between simulation curves and experiment data points.

Fig. 4 shows the forced vibration time history responses of the structure from the experiment. The time histories shown are for three different values of $r$ in Fig. 5 (b). The time history which shows successive peaks to be equal in magnitude is for $r=0.968$ (local peak observed at left of Fig. 5 (b)). The other two time histories are for $r=$ 0.991 and 1.008, which are some of the points near $r=1.0$ in Fig. 5 ( b ). It can be observed that these time histories show beating and that the beating period becomes shorter as $r$ goes beyond 1.0. Time histories from the experiment for forced vibration indicate that steady-state without beating occurs at and near a local peak while beating is observed away from these peaks.

Considering the effect of $e$ on the efficiency of the PID, experiment results show that the PID is most 
efficient for steel ball impacting with the steel wall $(e=0.37)$, followed by steel ball impacting with steel wall having $1.0 \mathrm{~mm}$ rubber lining $(e=0.46), 0.5 \mathrm{~mm}$ rubber lining $(e=0.58)$ and $3.0 \mathrm{~mm}$ cork lining $(e=$ $0.64)$, in that order. In terms of the $e$ values determined from the experiment, this shows that efficiency of the PID is highest for low $e$ values. Simulation results agree with this finding. An observation not possible in numerical simulation is that using a rubber lining over the steel confining walls substantially reduced noise during impacts ${ }^{(3)}$.

Although only a few examples are included due to space limitation, it should be noted that all simulations done (combinations of $\mu=0.54 \%, 1.29 \%, 2.52 \%, d=20,30,40 \mathrm{~mm}, r^{\prime}=0.9,1.0,1.1$, and $e=0.37$, $0.46,0.58,0.64)$ for comparison with the experiment results agree well ${ }^{133}$. It should also be noted that for very low $\beta$, the effect of the damper on the response of the structure is very dominant, but as $\beta$ increases, the effect becomes less dominant. Although experiments using $\beta$ 's representative of civil engineering structures were not done, it is deemed that the mathematical model and the computer program for simulation can accurately simulate structure responses when $\beta$ 's are representative of civil engineering structures. This is based on the fact that since they can accurately reproduce the results from experiment where $\beta$ is extremely small, i. e., dominant effect of the damper, then they should be more able to reproduce results for larger $\beta$ 's, i. e., less dominant effect of the damper.

\section{AMPLITUDE RATIO AND GAP RATIO}

The following ratios are best suited for quantifying damper efficiency for civil engineering purposes.

a) amplitude ratio, $X_{m}=x_{\max } / A_{\max }$ (Fig. 6) : ratio of the peak steady-state response amplitude of the main system with the damper obtained from a frequency sweep, over the peak steady-state response amplitude of the main system without the damper, $A_{\max }=\left(F_{0} / K\right) /(2 \beta)$. Compared with 1.0, the smaller $X_{m}$ is, the more effective is the damper.

The numerical procedure followed to determine $x_{\max }$ is as follows : simulation for 500 periods (natural period of the structure without damper) is done for each $r$ value. The steady-state response amplitude is determined from the root-mean-square of the simulation points from period 250 to period 500 and then multiplied by the square-root of two, i. e., assuming sinusoidal vibration. The reason for using the root-mean-square of the data points is because it has been observed from the experiments that structure response may be of the beating type (Fig.4), thus this method enables the comparison of amplitudes. Sample time histories from simulation showing the last 100 cycles (cycle 400 to 500) of structural response are shown in Fig. 7. ( a ) is for $r=0.995$ and shows equal peaks for successive cycles while ( $\mathrm{b}$ ) is for $r=$ 0.92 and shows beating. When the amplitude for each of the $r$ 's in the frequency sweep has been determined by the above procedure, they are then compared with each other and $x_{\max }$ is the maximum among the amplitudes for the frequency sweep.

b) gap ratio, $g=d / A_{\max }$ : ratio of the gap size over the peak steady-state response amplitude of the main system without the damper. The less sensitive $X_{m}$ is to $g$, the more reliable is the damper design.

\section{RESULTS OF NUMERICAL PARAMETRIC STUDY}

For this study, a frequency sweep from $r=0.9$ to 1.1 at intervals of 0.005 is considered. $\left(F_{0} / K\right)$ equal to 0.096 units is used. Gap size is a range from 1.0 to 50.0 units at intervals of 1.0 unit. The succeeding graphs (Figs. 8 to 11) are plotted with the horizontal axis as gap ratio and the vertical axis as amplitude ratio. (To reduce calculation time but still cover a wide range of gap ratio, $g$, resolution of $\Delta g=0.2$ is considered sufficient.) The significance of these axes definitions is that the lower the points are on the graph, the more efficient is the damper, and the longer the range of $g$ 's having nearly the same amplitude ratio, the less sensitive is the damper to variations in the amplitude of the excitation.

To get the average number of impacts per cycle (AIPC), the procedure is to count the total number of impacts that occur and divide this by the total number of cycles. The symbols plotted in the succeeding 
graphs which are marked as $<1$ IPC, 1 IPC, 2 IPC, and $>2$ IPC are actually rounded-off values of the AIPC, i. e., $<1$ IPC means AIPC $<0.5,1$ IPC means $0.5 \leqq \mathrm{AIPC}<1.5,2$ IPC means $1.5 \leqq \mathrm{AIPC} \leqq 2.5$, and $>2$ IPC means AIPC $>2.5$.

The general observations are that optimum performance occurs under 2 IPC, and efficiency drops when non-2 IPC condition prevails. Decrease in efficiency is gradual for change from 2 IPC to more than 2 IPC (3 or more impacts per cycle), but is drastic for change to less than 2 IPC.

\section{(1) Mass ratio}

Curves showing the relationship among $\mu, X_{m}$ and $g$ are given in Fig. 8. Except for the PID with $r^{\prime}>$ 1.0 , the general tend is for $X_{m}$ to decrease as $\mu$ increases. At the same time, the range of $g$ 's having 2 IPC becomes shorter, with the $g$ values becoming lower, as $\mu$ increases. For example, consider the curves in Fig. 8 (c) that show $g$ 's with 2 IPC. For $\mu=1.0 \%$, the range of $g$ is from 0.8 to 4.4 . When $\mu$ is increased to $3.0 \%$, it is from 0.4 to 1.6 . This range is shorter, with the $g$ values generally lower, than those for $\mu=1.0 \%$. It should be noted however, that the decrease in $X_{m}$ is generally not in linear proportion to the increase in $\mu$.

\section{(2) Coefficient of restitution}

Curves showing the relationship among $e, X_{m}$ and $g$ are given in Fig. 9. Consideration of Fig. 9 (a) for the CID, the graph indicates that increasing $e$ shifts the curves generally upwards. It should be noted however, that the lowest point among the curves does not necessarily fall on the curve with the lowest $e$ value. For example consider the points at optimum gap ratio, i. e., the respective lowest points of the three curves : when $e=0.25, X_{m}=0.32$; when $e=0.45, X_{m}=0.22$; and when $e=0.65, X_{m}=0.4$. This shows that although increasing $e$ from 0.25 to 0.45 produces a decrease in $X_{m}$, increasing $e$ further to 0.65 , causes an increase in $X_{m}$. This is because there is an optimum combination of $e$ and the rebound velocity after impact that gives maximum energy loss. The range of $g$ 's with 2 IPC generally shifts to the right, i. e., higher range when $e$ is increased. This shift is due to the higher velocity that the free mass possesses after each impact as compared with a lower $e$ value. The implication of the effects of $e$ as observed from Fig. 9 (a) is that maximum reduction in response amplitude is obtained for median values of $e$. Considering Fig. 9 ( $\mathrm{b}$ ) to ( $\mathrm{d}$ ), the major difference between the CID and the PID is that for the PID, increasing $e$ shifts the curves monotonically upwards, indicating a gradual reduction in efficiency as $e$ approaches 1.0. In other words, the efficiency of the PID increases as $e$ decreases.

\section{(3) Tuning of PID}

Graphs (b) to (d) of Figs. 8 and 9 show that the efficiency of the PID generally increases as $r^{\prime}$ approaches 0.0 (CID condition). Take for example the curves for $\mu=1.0 \%$ in Fig. 8. From Fig. 8 (a) for the CID, $X_{m}=0.32$ at optimum gap ratio. Similarly, at optimum gap ratio, $X_{m}=0.42$ from Fig. 8 ( b ) for PID with $r^{\prime}=0.5, X_{m}=0.5$ from Fig. 8 (c) for PID with $r^{\prime}=1.0$, and $X_{m}=0.8$ from Fig. 8 (d) for PID with $r^{\prime}=1.5$.

Considering the range of $g$ 's with 2 IPC, for $\mu=1.0 \%$, Fig. 8 ( b ) gives a range of 0.8 to 1.2 for $r^{\prime}=$ 0.5 , Fig. 8 (c) gives a range of 0.8 to 4.4 for $r^{\prime}=1.0$, and Fig. 8 (d) gives a range of 0.6 to 1.0 for $r^{\prime}=$ 1.5. This indicates that the range of $g$ with 2 IPC is longest for $r^{\prime}=1.0$ and decreases as $r^{\prime}$ differs from 1. 0 . This is because when the PID is tuned to the main system, it will be vibrating at its natural frequency when the main system is at resonance with the external excitation. Disregarding the limitation introduced by the length of the pendulum, it is possible for the PID to vibrate with large amplitudes enabling impacts to occur even for large $g$ 's, thus extending the upper limit where 2 IPC exists.

\section{(4) Effect of friction}

Material rusting is inevitable during the life of most civil engineering structures. Likewise, smoothness of the surface where the CID mass moves or where the PID mass is suspended from cannot be guaranteed. Thus friction will be present and studies $\left.{ }^{13)} \sim 15\right)$ have shown that friction is detrimental to the performance of the CID. Fig. 10 shows the performance of the CID without friction $(\nu=0.00)$ and with friction 
Amplitude ratio, $X_{m}$

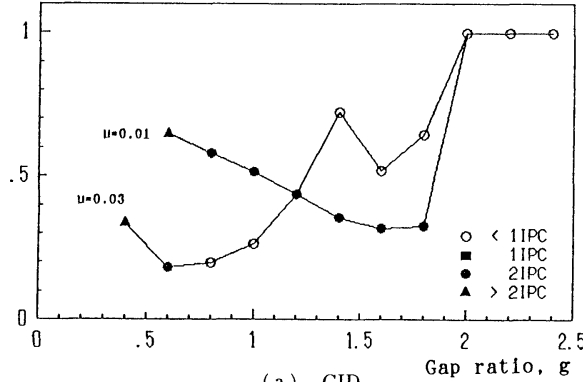

(a) CID

Amplitude ratio, $x_{m}$

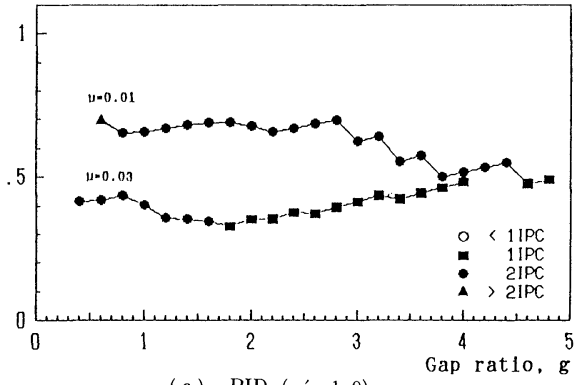

(c) PID $\left(r^{\prime}=1.0\right)$
Amplitude rat10, $X_{m}$

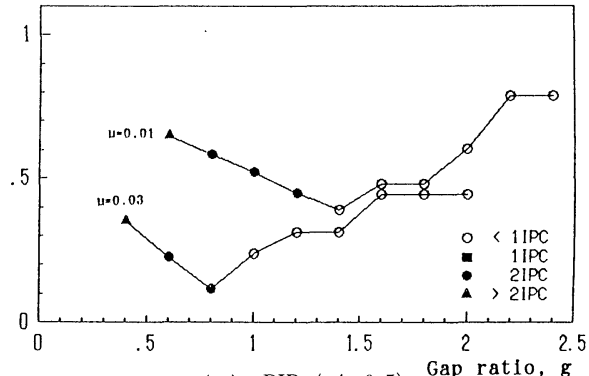

(b) PID $\left(r^{\prime}=0.5\right)$

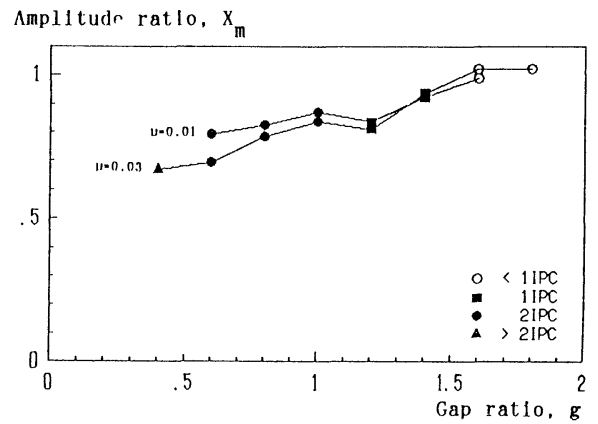

(d) PID $\left(r^{\prime}=1.5\right)$

Fig. 8 Effect of mass ratio $\left(\beta=0.0048, e=0.25, A_{\max }=10\right)$.

Amplitude ratio, $X_{m}$

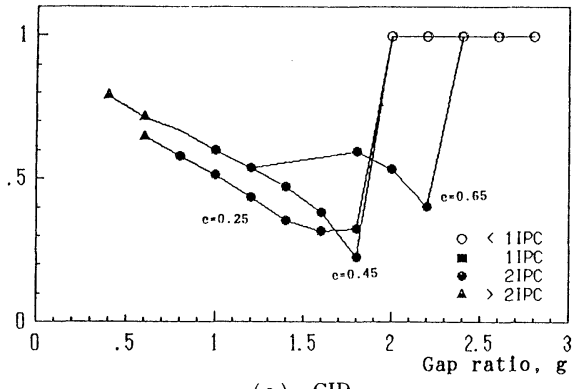

(a) CID

Amplitude ratio, $X_{\text {m }}$

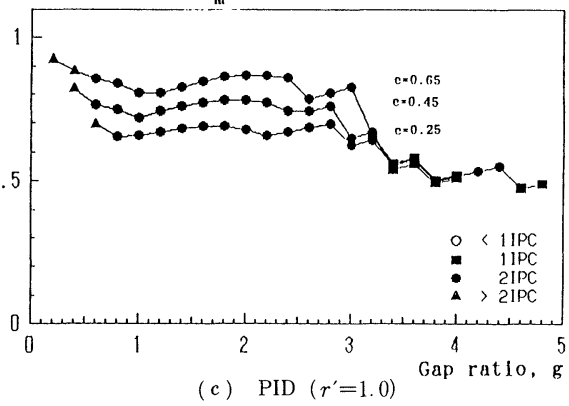

Amplitude ratlo, $X_{m}$

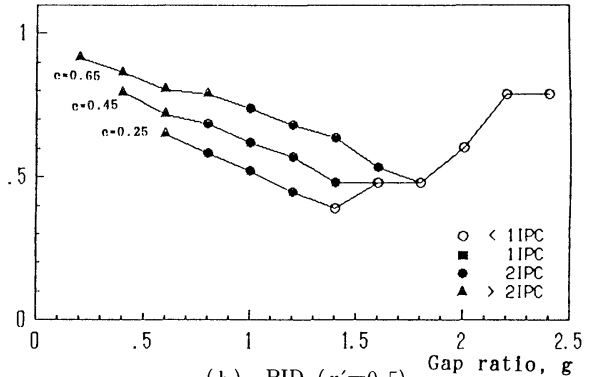

(b) PID $\left(r^{\prime}=0.5\right)$

Amplitude ratio, $x_{m}$

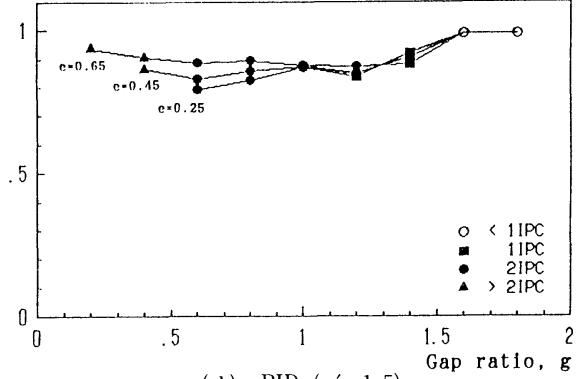

(d) PID $\left(r^{\prime}=1.5\right)$

Fig. 9 Effect of the coefficient of restitution $\left(\mu=0.01, \beta=0.0048, A_{\max }=10\right)$. 
coefficients $\nu=0.05,0.20$, and 0.50 . It is clear that friction reduces the efficiency of the CID. On the other hand, the PID could be made free from friction.

(5) Summary on the results of numerical parametric study

Results of the numerical parametric study indicate that the effect of $\mu$ on the performance of both the CID and the PID is approximately the same, unless the PID is too overtuned. On the other hand, a median value of $e$ will give better efficiency to the CID, while low $e$ is better for the efficiency of the PID. Under ideal conditions, the CID is more efficient than the PID, but the PID when tuned $\left(r^{\prime}=1.0\right)$ or undertuned $\left(r^{\prime}<1.0\right)$ close to 1.0 is much less sensitive to variations in the amplitude of the excitation. Non-ideal conditions like friction is shown to have a negative effect on the performance of the CID while the PID could be made practically free from friction.

\section{COMPARISON OF THE CID, PID AND TMD}

\section{(1) Comparison of efficiency}

For comparison, a main system having $\beta=0.0048$ and $\mu=$ $1.0 \%$ is utilized. Ideal conditions (no friction and imperfections) are assumed. Fig. 11 compares the performance of the CID, PID and TMD for a given set of parameters. For the CID and PID, $e=0.25$ while $g$ is varied. For the TMD, optimization is based on criteria given in Ref.16), i.e., determine condition where the two local peaks observed in a frequency sweep are equal in magnitude and are minimums. Tuning and TMD damping ratio giving this condition are the optimum values. For the main system and damper described, optimum TMD damping ratio is 0.06 and tuning is 0.99 . Response amplitude of main system with optimum TMD is plotted as the dashed-line. It is observed that optimum efficiency in terms of mass ratio is better for the TMD than for the CID or PID.

\section{( 2 ) Practical problems}

Regarding total performance, it is not only ideal efficiency that counts but also the relative ease of actually attaining such level of efficiency. The criteria could be determined theoretically, but physical conditions may pose limits on their practical implementation. Table 1 gives a summary and comparison of characteristics of the CID, PID, and TMD for suppression of horizontal motion.

Analyzing some of these constraints, consider for instance the space requirement characteristic. The available space in the main structure to locate the damper is often limited, thus it may not be possible to install a large mass $\mathrm{TMD}^{17)}$ or a large mass CID or PID. An alternative is to divide the single large mass into a number of smaller units having the same total mass with all other properties equal to that of the single large unit. Ref. 14) shows that dividing a large CID into a moderate number of smaller units (up to 9) gives the same level of performance. Free vibration experiments on the $\mathrm{PID}^{13)}$ show $\delta$ values for a given amplitude to be equal for a large PID compared with up to 4 smaller units. Space is required not only to accommodate the size of the damper, but also to allow the expected relative motion of that mass. Studies ${ }^{16) \sim 19)}$ have showed that the TMD displacement amplitude is often too large to be physically permissible. Thus the TMD damping ratio is increased to reduce its displacement. Similarly, the performance of the CID or PID is dependent on the gap size provided, but it may not be possible to provide 
Table 1 Comparison of Characteristics.

\begin{tabular}{|c|c|c|c|}
\hline Characteristics & CID & PID & TMD \\
\hline 1. implemented in civil engineering structures & no & no & yes \\
\hline 2. effectiveness for steady-state motion & moderate & moderate & high \\
\hline 3. complexity / maintenance requirements & low & low & moderate \\
\hline 4. durability & moderate & moderate & moderate \\
\hline 5. space requirement & moderate & moderate & high \\
\hline 6. sensitivity to tuning & $\sim \cdot \sim$ & low & high \\
\hline 7. sensitivity to damping of the damper & $\sim \sim$ & $\sim \cdot \sim$ & high \\
\hline 8. importance of the location of the damper & high & high & high \\
\hline 9. sensitivity to amplitude of the excitation & high & moderate & low \\
\hline 10. material dependence (coefficient of restitution) & moderate & moderate & moderate \\
\hline
\end{tabular}

* obtained from Ref. 19)

the design gap size. For both the TMD and the impact dampers, modifying their properties to suit the available space leads to performance lower than theoretically possible.

Consider tuning and damping of the damper. It is known that the TMD is sensitive to these two properties, and that proper tuning and provision of the correct TMD damping ratio is difficult to attain under civil engineering conditions. On the other hand, the CID does not have these properties while the PID has only the tuning property. It has been shown that the PID is insensitive to tuning as long as it is undertuned. In fact the performance improves as tuning approaches zero.

\section{CONCLUDING REMARKS}

A semi-theoretical study has been done on the classical impact damper (CID) and the pendulum impact damper (PID) considering parameter ranges deemed representative of most civil engineering structures.

Experiment to verify the validity of the mathematical model for the PID shows that good agreement exists between simulation and experiment results.

Numerical parametric study indicates that both impact dampers can be used to effectively control horizontal vibrations due to harmonic exciting force. The advantage of the CID over the PID is that for a given mass ratio, the CID is more efficient under ideal conditions. On the other hand, the PID is superior to the CID in that it is not sensitive to variations in the amplitude of the excitation when tuned or undertuned close to the structural frequency. This is exemplified by the flat curves shown in Figs. 8 (c) and 9 (c) for a long range of gap ratio.

A comparison with the TMD under both ideal conditions and with practical constraints is provided. The comparison shows that the TMD is most efficient among the three dampers when ideal conditions are considered, but under more realistic conditions, the CID and the PID provide certain advantages: e. g. , damping of damper and tuning parameter are irrelevant to the CID, and undertuning is not detrimental to the PID.

Finally, although not included in this paper, design equations ${ }^{13)}$ for the CID and PID have been developed to eliminate most of the lengthy and cumbersome computations necessary due to the nonlinearity introduced by the impacts.

\section{REFERENCES}

1) Lieber, P. and Jensen, D. P. : An Acceleration Damper : Development, Design, and Some Applications, Transactions of the ASME, Vol.67, pp. 523-530, 1945.

2) Masri, S. F. and Caughey, T. K. : On the Stability of the Impact Damper, Journal of Applied Mechanics, Transactions of the ASME, Vol. 88 (Series E), pp. 586-592. 1966.

3) Reed III. W. H. : Hanging-Chain Impact Dampers: A Simple Method for Damping Tall Flexible Structures, Wind Effects on Buildings and Structures, Paper 36, pp. 284-321, 1967.

4) Bapat, C. N. and Sankar, S. : Single Unit Impact Damper in Free and Forced Vibration, Journal of Sound and Vibration, Vol. 99. No. 1, pp. 85-94, 1985. 
5) Bapat, C. N., Popplewell, N. and McLachlan, K. : Stable Periodic Motions of an Impact-Pair, Journal of Sound and Vibration, Vol. 87. No. 1, pp. 19-40, 1983.

6) Nigm, N. M. and Shabana, A. A. : Effect of an Impact Damper on a Multi-Degree of Freedom System, Journal of Sound and Vibration, Vol. 89, No. 4, pp.541-557, 1983.

7) Popplewell, N., Bapat, C. N. and McLachlan, K. : Stable Periodic Vibroimpacts of an Oscillator, Journal of Sound and Vibration, Vol.87, No.1, pp.41-59, 1983.

8) Sadek, M. M. and Mills, B. : Effect of Gravity on the Performance of an Impact Damper : Part 1. Steady-State Motion, Mechanical Engineering Science Journal, Vol. 12, No.4, pp.268-277, 1970.

9) Thomas, M. D. and Sadek, M. M. : The Effectiveness of the Impact Damper with a Spring-Supported Auxiliary Mass, Mechanical Engineering Science Journal, Vol.16, No.2, pp. 109-116, 1974.

10) Araki, Y., Yokomichi, I. and Jinnouchi, Y. : Impact Damper with Granular Materials (4 th report, Frequency Response in a Horizontal System), Bull of JSCE, Vol.29, No. 258, pp. 4334-4338, Dec. 1986.

11) Izumi, U., Yamada, K. and Watanabe, $T_{*}$ : Effectiveness of Chain Damper in Suppressing Wind-Induced Vibration of Illumination Post (Real Structure Vibration Experiment), Proceedings of the 43 rd Annual Conference of JSCE, Vol. 1, Paper 308, pp. 668-669, 1988 (in Japanese).

12) Jo, I., Kaneko, T., Nagatsu, S., Takahashi, C. and Kimura, M. : Development of Highway Lighting Pole with Resistance to Wind Vortex-Induced Oscillations, Kawasaki Seitetsu Giho, Vol. 20, No.4, pp.58-64, 1988 (in Japanese).

13) Chua, G. S. : A Study On Classical Impact Damper And Pendulum Impact Damper For Civil Engineering Application, Doctoral Dissertation. University of Tokyo, June 1989.

14) Bapat, C. N. and Sankar, S. : Multiunit Impact Damper: Re-examined, Journal of Sound and Vibration, Vol. 103 , No. 4. pp. 457-469, 1985.

15) Mansour, W. M. and Teixeira Filho, D. R. : Impact Dampers with Coulomb Friction, Journal of Sound and Vibration, Vol. 33. No. 3, pp. 247-265, 1974.

16) Warburton, G. B. and Ayorinde, E. O. : Optimum Absorber Parameters for Simple Systems, Earthquake Engineering and Structural Dynamics, Vol. 8, pp. 197-217, 1980.

17) Leipholz, H. H. E. (editor), Petersen, N. R. (author) : Design of Large Scale Tuned Mass Dampers, Structural Control, North-Holland Publishing Company and SM Publications, IUTAM, pp.581-596, 1980.

18) Fujino, Y. . Warnitchai, P. and Ito, M. : Suppression of Galloping of Bridge Tower Using Tuned Mass Damper, Journal of the Faculty of Engineering (B), University of Tokyo, Vol. 38, No. 2, pp.49-73, 1985.

19) Wiesner, K. B. : Taming Lively Buildings, Civil Engineering. pp. 54-57, June 1986.

(Received July 10 1989) 